Revue européenne des migrations internationales

vol. $27-n^{\circ} 1 \mid 2011$

La construction de l'altérité dans l'espace noir atlantique : Etats-Unis - France - Caraïbes - Amérique latine

\title{
Laurence ROULLEAU-BERGER, Migrer au féminin
}

\section{Adelina Miranda}

\section{(2) OpenEdition \\ Journals}

Édition électronique

URL : https://journals.openedition.org/remi/5416

DOI : $10.4000 /$ remi. 5416

ISSN : $1777-5418$

\section{Éditeur}

Université de Poitiers

Édition imprimée

Date de publication : 1 juin 2011

Pagination : 181-182

ISBN : 979-10-90426-00-9

ISSN : 0765-0752

\section{Référence électronique}

Adelina Miranda, «Laurence ROULLEAU-BERGER, Migrer au féminin », Revue européenne des migrations internationales [En ligne], vol. 27 - n¹ | 2011, mis en ligne le 30 août 2011, consulté le 15 avril 2022. URL : http://journals.openedition.org/remi/5416 ; DOI : https://doi.org/10.4000/remi.5416

Ce document a été généré automatiquement le 15 avril 2022.

(c) Université de Poitiers 


\title{
Laurence ROULLEAU-BERGER, Migrer au féminin
}

\author{
Adelina Miranda
}

\section{RÉFÉRENCE}

Laurence ROULLEAU-BERGER, Migrer au féminin,Paris, P.U.F, 2010, 192 p., ISBN :

978-2-13-057745-4.

1 Comme Laurence Roulleau-Berger le souligne en ouverture de cet ouvrage, le migrant, et plus précisément la migrante, est une figure emblématique des transformations des ordres économiques locaux et globaux. Analysé à travers ce prisme, le processus de la féminisation des migrations dévoile tant les liens associés à «la constitution d'un marché du sous-emploi au cœur duquel se trouve la figure de la femme en migration contrainte par un marché du travail globalisé » (p. 79) que la pluralité de situations migratoires féminines. Sur des routes de plus en plus diversifiées, «la figure de la migrante ", souvent représentée au singulier, se décompose, pour laisser place à une singularité plurielle, agencée entre appartenances nationales, qualifications, niveaux d'études et la capacité à s'adapter à un marché du travail segmenté, à une économie polycentrée, aux formes de pluralisation et de multipolarisation des économies. En s'appuyant sur une recherche effectuée de 2003 à 2006 cofinancée par la DARES, le GED et le Fonds social européen (Nouvelles migrations internationales, genre et travail: inscriptions économiques, discriminations et histoires de femmes d'Afrique subsaharienne, du Maghreb, d'Europe centrale et orientale et de Chine continentale en France), l'auteure explique que les diversifications ne sont pas seulement la conséquence des phénomènes structuraux (économiques, politiques, etc.) mais également des capacités du sujet féminin de combiner et agencer ces éléments avec leurs expériences. Les migrantes s'inscrivent ainsi dans un «espace pluridimensionnel » disposé entre les pôles des mono-migrations internationales et des pluri-migrations transnationales; confrontées aux dominations sociales, ethniques et sexuelles, elles se trouvent engagées dans des 
formes de travail différenciées et inégalement légitimées économiquement où se combinent hiérarchisation et sexuation du marché du travail.

2 Le livre se compose de quatre parties. Dans le premier chapitre (Circulations et diversité des routes migratoires), l'auteure souligne comment «les formes d'engagements dans le travail restent largement influencées par les histoires politiques et sociales des marchés du travail du pays d'origine » (p.62). Les carrières migratoires sont autant déterminées par les manières dont les sujets mobilisent savoirs et apprentissages transmis dans la famille que par les savoirs sociaux, techniques et professionnels. Et pourtant, malgré la pluralité des distinctions, au bout de leur chemin, les migrantes vivent une déqualification - qui peut prendre les formes de monoactivité, pluriactivité, polyactivité - qui leur demande une forte capacité adaptative. Dans le second chapitre (Institutions économiques et grammaires de l'injustice), elle analyse les inégalités socioéconomiques vécues par les migrantes (exploitation, marginalisation économique, injustice de type culturel ou symbolique) en tenant compte du fait que les modes d'accès à l'emploi sont sexués et ethnicisés. Les migrantes vivent souvent une imposition de l'inactivité, des fortes contraintes à l'accès au travail régulier et se retrouvent dans des circuits informels d'autant plus que le diplôme et le niveau d'étude protègent peu les femmes immigrées du chômage. C'est comme si «l'expérience migratoire contenait une dette qui devait se payer par l'acceptation de situations doublement disqualifiantes » (p. 85). Dans le troisième chapitre (Dispositifs économiques polycentriques), l'auteure approfondit les dispositifs économiques qui se mettent en place sur les routes migratoires. Ces champs économiques se construisent à travers des assemblages d'activités et d'espaces qui contiennent des hiérarchies qui assignent des places et des rôles aux femmes au sein de division sociale, ethnique et sexuelle du travail. Et pourtant, malgré la prépondérante assignation des femmes dans des " enclaves ethniques ", notamment pour celles qui vivent en insécurité linguistique, ou dans les «niches ethniques» (nettoyage, restauration et hôtellerie, secteur agricole), certaines femmes migrantes arrivent à mettre en place de dispositifs intermédiaires qui leur permettent de s'investir dans le commerce ou dans la petite production urbaine. Le chapitre quatre (Cosmopolitation des biographies) permet de saisir la diversification des biographies. Ce processus lié à la pluralisation et la hiérarchisation des espaces économiques globaux et locaux englobe différentes formes de cosmopolisation qui s'élaborent tant «par le bas » que « par le haut ». La question du travail des femmes en migration joue donc un rôle central car elle relève la complexité de la modernité avec ses injonctions contradictoires : travailler et passer d'un emploi précaire à l'autre, développer des compétences adaptatives et vivre un constant brouillage des ordres normatifs. Les migrantes sont prises dans un jeu mondialisé qui oscille entre la propriété et la perte de soi, estime et honte, altérisation du soi, possibilité de perte de la capacité réflexive, appartenance à des classes dénationalisées. Enjeux économiques et dynamiques culturels se rencontrent : les diffractions du travail se structurent sur des « régimes d'altérité » (qui peuvent être faible, forte ou partiellement autonome) qui deviennent des principes qui légitiment les conditions de travail subalterne.

En mettant en exergue comment les parcours migratoires des femmes se placent au centre des différentes configurations migratoires (multipolaires, transnationales et pendulaires), cet ouvrage permet de "comprendre comment se hiérarchisent les spatialités et comment ces hiérarchies sont régulièrement bousculées, comment les individus sont contraints à des formes de multi-appartenances, comment des réseaux 
transnationaux et réticulaires se forment au-dessus des États-nationaux en créant des processus de conjoint et de disjonction culturels, économiques et symboliques » (p. 10). Par ailleurs, en mettant constamment en relation trois niveaux d'analyse (espace, marché du travail et capital), Laurence Roulleau-Berger démontre que les inégalités sociales, ethniques et de genre se multiplient mais que, en même temps, les populations y sont différemment exposées. Cette lecture multiscalaire des migrations indique comment les grammaires de l'injustice se pluralisent tout en se hiérarchisant selon les contextes locaux et globaux en fonction de traditions culturelles, des ordres institutionnels et des identités résultant des processus de socialisation.

4 L'étude relève ainsi que les parcours migratoires sont liés tant au capital spatial (qui peut apparaître tantôt comme positif, tantôt comme négatif, tantôt comme une ressource, tantôt comme une contrainte) qu'au capital social (qui se construit différemment en fonction des contextes de départ, des pratiques économiques, de la valeur des biens mis en circulation, de la nature des savoir-faire, des qualifications, des compétences). Mais, dans les situations migratoires peuvent se développer des stratégies individuelles et collectives de résistance qui portent à l'affirmation d'autres formes de globalisation non hégémoniques ou bien éloignées d'une globalisation capitalistes. On sera attentif notamment à l'importance de la «bifurcation», cet espace-temps où se produisent des changements de régimes spatiaux et économiques dans l'organisation des itinéraires des migrantes. Les Sujets au féminin semblent alors s'affirmer dans une tension philosophique et éthique qui peut donner vie à de nouvelles formes de mondialisation. Laurence Roulleau-Berger montre que l'individuation globalisée vécue par les femmes migrantes incite à repenser le processus d'individuation car il se situe dans une multiplicité de lieux et donc d'échelles de pouvoir. Ce phénomène, décrit comme étant multi-facette, hétérogène et en constant devenir, permet alors de repenser les formes d'assignation économique et culturelle féminines. Et, en conséquence, l'étude des migrations féminines finit par interroger les dispositifs économiques, les formes éthiques et les processus d'émancipation de la société sédentaire.

\section{AUTEURS}

\section{ADELINA MIRANDA}

Faculté de Sociologie, Naples, CRESPPA, Paris, MIGRINTER, Poitiers 